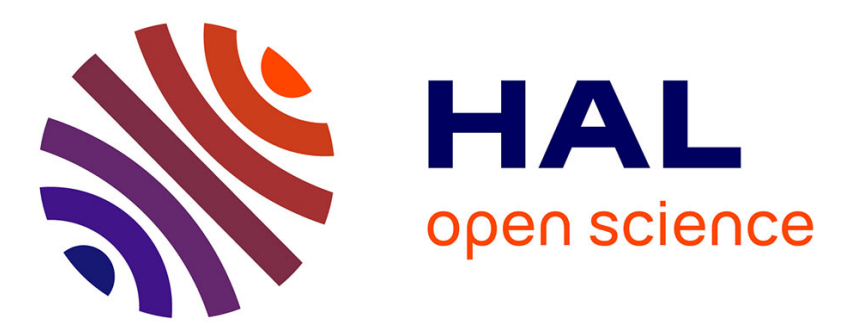

\title{
Thermochemical Interactions Versus Site Competition in Grain Boundary Segregation and Embrittlement in Multicomponent Systems
}

\author{
M. Guttmann
}

\section{- To cite this version:}

M. Guttmann. Thermochemical Interactions Versus Site Competition in Grain Boundary Segregation and Embrittlement in Multicomponent Systems. Journal de Physique IV Proceedings, 1995, 05 (C7), pp.C7-85-C7-96. 10.1051/jp4:1995707 . jpa-00254000

\author{
HAL Id: jpa-00254000 \\ https://hal.science/jpa-00254000
}

Submitted on 1 Jan 1995

HAL is a multi-disciplinary open access archive for the deposit and dissemination of scientific research documents, whether they are published or not. The documents may come from teaching and research institutions in France or abroad, or from public or private research centers.
L'archive ouverte pluridisciplinaire HAL, est destinée au dépôt et à la diffusion de documents scientifiques de niveau recherche, publiés ou non, émanant des établissements d'enseignement et de recherche français ou étrangers, des laboratoires publics ou privés. 


\title{
Thermochemical Interactions Versus Site Competition in Grain Boundary Segregation and Embrittlement in Multicomponent Systems
}

\author{
M. Guttmann \\ IRSID, Voie Romaine, BP. 320, 57214 Maizières-Les-Metz cedex, France
}

\begin{abstract}
Several elementary mechanisms play a role in intergranular segregation and embrittlement in multicomponent systems : site competition, attractive and repulsive chemical interactions. The physical significance of these interactions, their thermodynamic modeling and validity, and the experimental evidence available for each of them are dicussed with particular emphasis on Fe-base alloys with metallic and non-metallic segregating solutes.
\end{abstract}

\section{INTRODUCTION}

Although sometimes still underrated, intergranular segregation is now recognized as a determining factor in materials properties and behaviour during fabrication or service of engineering materials. In the past, these phenomena have been studied in the complex, multicomponent and often multiphase real materials themselves (e.g. steels), but also in simplified binary and ternary model alloys of intermediate or ultrahigh purity. With the development of modern steelmaking processes and the emergence of very low alloy steels such as IFS and steels with "very low everything", the ultrahigh purity alloys have ceased to be model materials only. Therefore the mechanisms identified in them are now of direct technological interest.

Pure metals have of ten revealed the only dependable means for identifying and elucidating the precise elementary mechanisms of segregation and embrittlement. Conversely, care must be taken when interpreting the properties of complex real materials in the light of results obtained with simpler ultra-pure alloys. This is the case, in particular, for interactive and/or competitive segregation to grain boundaries. A number of experimental results have shown that the segregation of several components are almost never independent of each other, as some elements enhance while others impede the segregation of a given species. The complex behaviours observed have been concurrently attributed to chemical interactions at the interface and in the bulk, or to competition for atomic interfacial sites between the various segregating species. Of particular importance is the case of iron and steels, in which some non-metallic solutes (e.g. P, S) embrittle while others (e.g. C, B) de-embrittle the grain boundaries, and in which most metallic additions ( $\mathrm{Mn}, \mathrm{Ni}, \mathrm{Cr}, \mathrm{Mo}, \mathrm{Ti})$ also play a role in embrittlement processes. Considerable experimental and theoretical efforts have been devoted to these phenomena due to their technological importance.

In view of the wealth of data and of their somewhat conflicting interpretations over the last twenty years, a discussion of this problem seemed necessary. The present paper more specifically focusses on the elementary mechanisms involved in interfacial segregation. The exemplary case of reversible temper embrittlement (RTE) of low-alloy steels and martensitic stainless steels will be only briefly discussed here in passing, since it is treated at length elsewhere [1].

\section{ELEMENTARY MECHANISMS INVOLVED IN INTERFACIAL SEGREGATION}

\subsection{Binary solutions.}

The basic equation describing the segregation of a single solute $\boldsymbol{i}$ at an interface $\boldsymbol{\Phi}$ in a solvent $\boldsymbol{A}$ is the well known McLean isotherm, eqn la (table 1) [2]. In the absence of chemical interactions between atoms i.e. when both the matrix and the interface are considered ideal (or Henryan) - the segregation energy is constant or, more generally, depends only on temperature, eqn 2 (table 1). As shown by Fowler, the mutual interactions between atoms in the binary systems can strongly modify the segregation pattern [3]. Using the very simple regular solution approximation introduces a chemical interaction term in the segregation energy eqn 3 (table 1). In particular when the solute-solute interaction is attractive with respect to the solvent, $\alpha_{\mathrm{ii}}$ is negative and segregation is enhanced. In this case, unmixing of a solute-rich $2 \mathrm{D}$ "phase" is expected ultimately at the interface. Conversely, when the relative solute-solute interaction is 
repulsive $\left(\alpha_{\mathrm{ii}}^{\Phi}>0\right)$ the segregation is lowered. The segregation energy being inversely related to the solubility limit [4], segregation and precipitation (or unmixing) reflect the same basic thermodynamic properties of the solution, among which the mutual chemical interactions play a determining role.

\begin{tabular}{|l|l|l|}
\hline $\mathrm{X}_{\mathrm{i}}^{\Phi}=\frac{\mathrm{X}_{\mathrm{i}}^{\mathrm{B}} \exp \left(\Delta \mathrm{G}_{\mathrm{i}} / \mathrm{RT}\right)}{1+\mathrm{X}_{\mathrm{i}}^{\mathrm{B}} \exp \left(\Delta \mathrm{G}_{\mathrm{i}} / \mathrm{RT}\right)}$ & {$[1 \mathrm{a}]$} & $\begin{array}{l}\text { Basic equation for binary and } \\
\text { ternary** segregation } \\
\text { without competition for sites }\end{array}$ \\
\hline $\mathrm{X}_{\mathrm{i}}^{\Phi}=\frac{\mathrm{X}_{\mathrm{i}}^{\mathrm{B}} \exp \left(\Delta \mathrm{G}_{\mathrm{i}} / \mathrm{RT}\right)}{1+\mathrm{X}_{1}^{\mathrm{B}} \exp \left(\Delta \mathrm{G}_{1} / \mathrm{RT}\right)+\mathrm{X}_{2}^{\mathrm{B}} \exp \left(\Delta \mathrm{G}_{2} / \mathrm{RT}\right)}$ & {$[1 \mathrm{~b}]$} & $\begin{array}{l}\text { Basic equation for ternary } \\
\text { segregation } \\
\text { with competition for sites }\end{array}$ \\
\hline$\Delta \mathrm{G}_{\mathrm{i}}=\mathrm{Q}_{\mathrm{i}}^{\circ}=$ constant or : & $\begin{array}{l}{[2 \mathrm{a}]} \\
{[2 \mathrm{~b}]}\end{array}$ & $\begin{array}{l}\text { Segregation energy for } \\
\text { ideal or Henryan } \\
\text { grain boundary }\end{array}$ \\
$\Delta \mathrm{G}_{\mathrm{i}}=\Delta \mathrm{G}_{\mathrm{i}}^{\circ}=\Delta \mathrm{H}_{\mathrm{i}}^{\circ}-\mathrm{T} \Delta \mathrm{S}_{\mathrm{i}}^{\circ}$ & {$[3]$} & $\begin{array}{l}\text { Segregation energy for } \\
\text { binary regular grain boundary }\end{array}$ \\
\hline$\Delta \mathrm{G}_{\mathrm{i}}=\Delta \mathrm{G}_{\mathrm{i}}^{\circ}-2 \alpha_{\mathrm{ii}}^{\Phi} \mathrm{X}_{\mathrm{i}}^{\Phi}$ & {$[4 \mathrm{a}]$} & $\begin{array}{l}\text { Segregation energy for } \\
\text { ternary regular grain boundary }\end{array}$ \\
\hline$\Delta \mathrm{G}_{\mathrm{i}}=\Delta \mathrm{G}_{\mathrm{i}}^{\circ}-2 \alpha_{\mathrm{i}}^{\Phi} \mathrm{X}_{\mathrm{i}}^{\Phi}+\alpha_{\mathrm{ij}}^{\prime} \mathrm{X}_{\mathrm{j}}^{\Phi} \quad$ More generally : & {$[4 \mathrm{~b}]$} \\
$\Delta \mathrm{G}_{\mathrm{i}}=\left(\Delta \mathrm{H}_{\mathrm{i}}^{\circ}-\mathrm{T} \Delta \mathrm{S}_{\mathrm{i}}^{\circ}\right)-2 \alpha_{\mathrm{i}}^{\Phi} \mathrm{X}_{\mathrm{i}}^{\Phi}+\alpha_{\mathrm{ij}}^{\prime} \mathrm{X}_{\mathrm{j}}^{\Phi}$ & &
\end{tabular}

Table 1 - Basic types of expressions for the segregation equations and energies.*

* All these equations can be derived using either the classical thermodynamic formalism along the lines of Defay and Prigogine [5] or the statistical mechanics approach originally used by McLean [2] and Fowler. [3].

In eqns $1 \mathrm{a}$ and $1 \mathrm{~b}$, the denominators have been simplified by omitting the generally negligible terms $-X_{i}^{B}$,

** In ternary non-competitive segregation, $\mathrm{X}_{i}^{\Phi}$ is the occupancy ratio in the intergranular "sub-lattice" of $\mathrm{i}$ atoms.

\subsection{Ternary systems}

In ternary systems the situation is much more complex.

2.2.1 Site competition. The first factor of complexity arises from the possibility of competition for sites at the interface between the segregating species. In that case, the segregations are given by eqns $1 \mathbf{b}$. The more surface active element (that with higher $\Delta \mathrm{G}_{\mathrm{i}}^{\circ}$ ) preferentially occupies the grain boundary sites available for segregation, thus preventing the other element from segregating. This argument has repeatedly been used to explain a number of antagonistic segregation behaviours. However, the phenomenon is much more complex and alternative or additional mechanisms must be considered.

First of all, site competition effects should in principle be efficient only at relatively high coverages. Nevertheless, they are also invoked for quite low coverages of both species [6-10]. Furthermore, heed has generally not been paid to the real sites occupied by segregating atoms. It is interesting to notice that some of the largest antagonistic segregation effects generally explained in terms of site competition actually take place between atoms like $\mathrm{C}$ or $\mathrm{B}$, and $\mathrm{P}$ or $\mathrm{S}$, which do not occupy the same type of sites in the matrix, the first being interstitial and the others substitutional. In spite of the distorted and much more complex structure of the grain boundaries than of the crystal, the considerable size difference between these two classes of atoms certainly causes them to be accomodated in different intergranular sites at the lower coverages. ${ }^{1}$ In addition, molecular dynamics calculations performed for $\Sigma 5$ and $\Sigma 9$ boundaries in $\alpha$ - Fe have shown that, as saturation is approached, the segregation of the former type of atoms leaves the boundary structure virtually unaltered and undistorted, whereas that of the latter species completely changes the intergranular atomic configuration and leads to considerable elastic stresses [11]. In ternary systems, therefore, the real site occupancy pattern and atomic structure of the grain boundaries containing both types of segregated solutes will necessarily be much more complex than can be predicted on the basis of the simplistic site competition argument. The site competition hypothesis may therefore not be sufficient to explain all the effects ascribed to it and other phenomena have to be invoked. This complexity will be multiplied by the variety of coexisting grain boundary structures, which leads to an extremely wide range of segregations of both solutes on individual grain boundary facets [12].

\footnotetext{
1 In the case of the free surface, however, this purely steric arguments does not hold and quite different types of atoms can occupy, and therefore compete for, the same sites.
} 
Last but not least, in many systems, although competitive segregation would be expected given the size of the two solute atoms and the similar nature of the matrix sites occupied by them, an opposite behaviour is observed : not only the segregations of both substitutional elements do not hinder each other but they even increase when the concentration of one of them is increased (figs I and 2). Obviously, a different and stronger mechanism counteracts the effect of site competition.

2.2.2. Chemical interactions. In general, the solutions where segregation takes place are far from ideal, that is, the atoms chemically interact with each other in the bulk. This implies that they will do so at the grain boundary as well. This evidence will provide us with some clues for solving the above-mentioned contradictions. In order to apprehend the effect of chemical interactions on the segregation process, the simple formalisms developed by Fowler [3] and by Defay and Prigogine [5] for binary alloys, which simply consist in considering the activities instead of the concentrations both in solution and at the interface, have been extended to ternary systems, leading to a more general expression of the segregation free energy (eqn 4 , table, 1) [13-15]. In a simplified form where only ternary chemical terms are explicited, it reads :

$$
\Delta \mathrm{G}_{\mathrm{i}}=\Delta \mathrm{G}_{\mathrm{i}}^{\circ}+\alpha_{\mathrm{ij}}^{\prime} \Phi \mathrm{X}_{\mathrm{j}}^{\Phi}
$$

The interesting feature here is the ternary chemical term $\alpha^{\prime} \Phi_{i j} X_{j}^{\Phi}$ arising from the interaction energy balance between the atoms of the three species: the two solutes $(i, j)$ and the solvent. ${ }^{2}$

- Preferential attractive interactions between $i$ and $j\left(\alpha_{i j}^{\prime}>0\right)$ enhance the segregation of both solutes with respect to those in the two binary systems. When the $\mathrm{i}-\mathrm{j}$ affinity or the bulk concentrations are increased, or when the temperature is lowered, the interaction may lead to the unmixing of a (i+j)-rich 2-D compound. This is probably the case at the upper plateaus in figs 1 and 2 which correspond to the saturation of the grain boundary and surface, respectively [16]. When the attractive $i-j$ interaction is sufficiently high, the more concentrated solute may eventually trap the minor constituent in the matrix in the form of atomic pairs, clusters or precipitates, thus preventing it from segregating to the interfaces $[17,18]$. The two processes, enhanced co-segregation and gettering, can thus compete according to a complex pattern which depends on the intensity of the $i-j$ interaction, temperature, concentrations of $i$ and $j$, and on the effects of other elements on both of these two processes [19].

- Conversely, a repulsive interaction between $i$ and $j\left(\alpha_{i j}^{\prime}<0\right)$ causes the species with the lower intrinsic segregation energy to segregate less or even to de-segregate in the presence of the other [13-15].

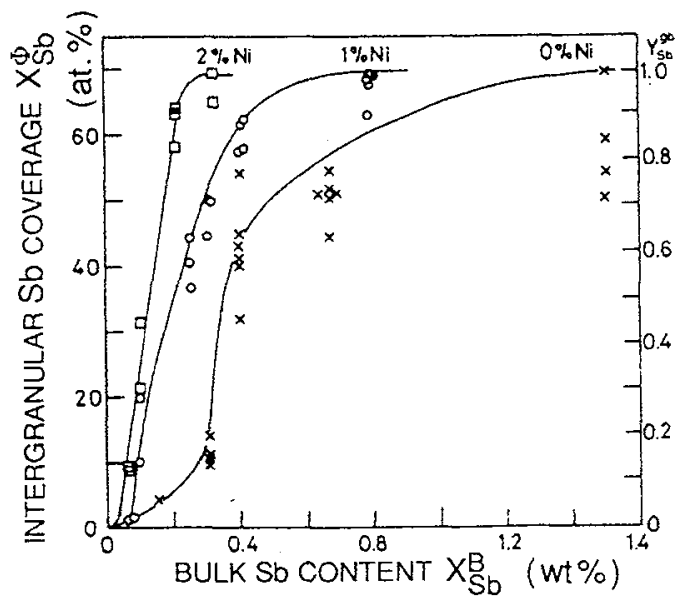

Fig. 1 - Influence of $\mathrm{Ni}$ additions on $\mathrm{Sb}$ intergranular segregation in high purity $\mathrm{Fe}$ at $550^{\circ} \mathrm{C}$. [21]

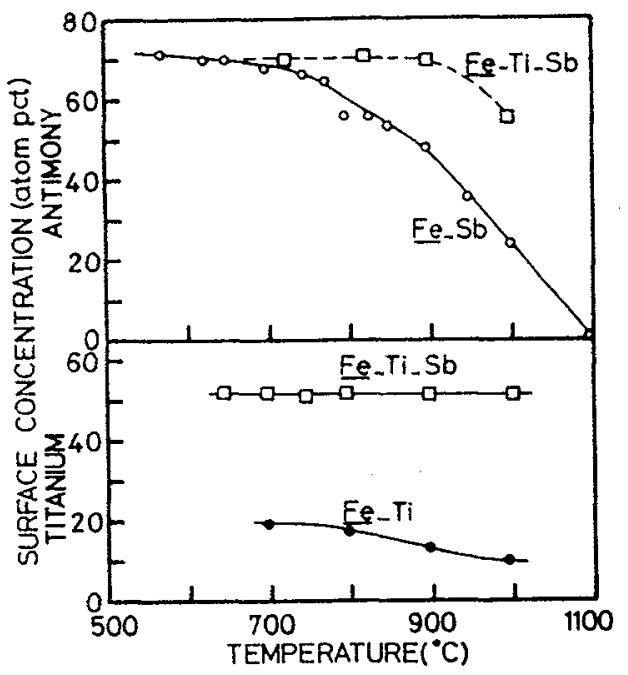

Fig. 2 - Mutual influence of Ti and Sb on each other's segregation at free surface of high purity Fe. [22]

\footnotetext{
2 As described in detail in the original publications [13-15], these interaction terms exclusively arise from activity coefficients. It is therefore suffocating to read the claim that the activity arguments "do not support the interaction model" [24] !
} 


\section{EXPERIMENTAL EVIDENCE FOR INTERACTIVE SEGREGATION}

\subsection{Binary solutions.}

The Fowler types of behaviour are illustrated by the grain boundary segregation of $\mathrm{Sb}$ and $\mathrm{P}$ in $\mathrm{Fe}$, respectively (fig. 3). The former case indicates relative $\mathrm{Sb}-\mathrm{Sb}$ attraction, while the P-P interaction appears weakly repulsive. The latter results have been interpreted by their authors as due to the effect of the segregation entropy rather than of chemical interactions, and a good correlation of the results with eqn $2 \mathrm{~b}$ is effectively obtained [7]. However when all the results are used for this correlation, as it si the case in fig. $3 \mathrm{~b}$, the scatter is comparable. On the other hand, the $\mathrm{Sb}$ results on fig. $3 \mathrm{a}$ being obtained for a single temperature by varying the bulk $\mathrm{Sb}$ content only, cannot be explained by an effect of $\Delta S_{i}$. In any event, it appears already for the simple case of binary alloys that mere numerical correlations do not identify the physical mechanism with certainty, as each correlation can appear as good... or as bad as the other [18,20].
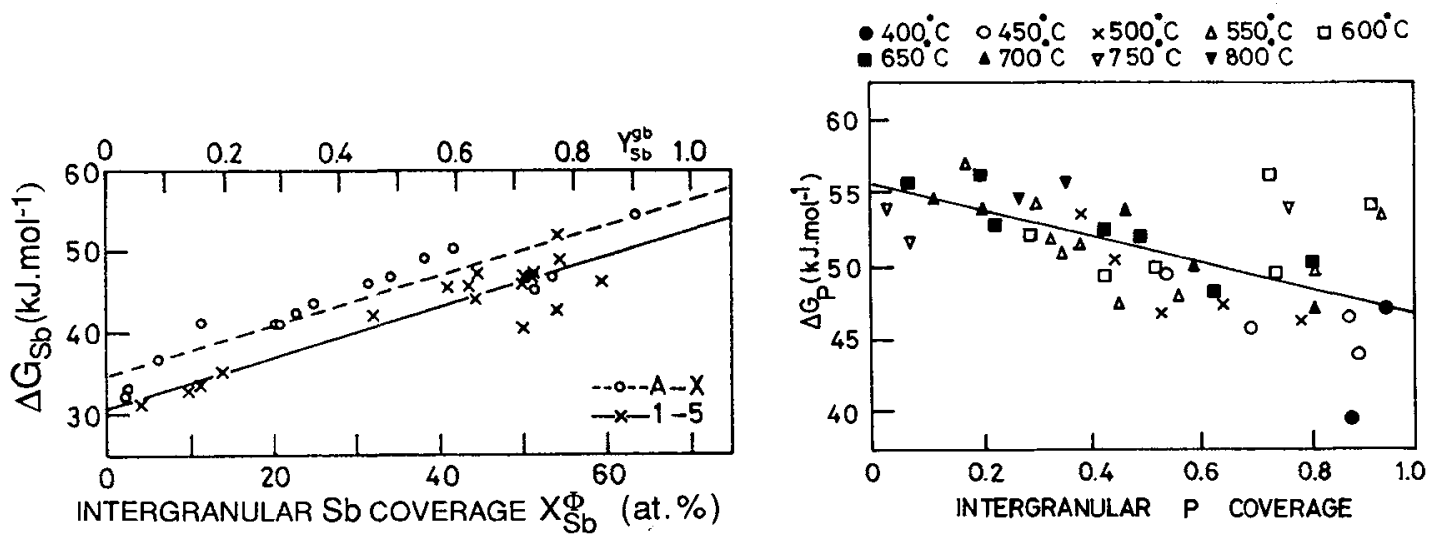

Fig. 3 - Linear correlations between segregation free energies of Sb (a) [21] and $\mathrm{P}$ (b) [18] and their own respective intergranular coverage. (Original results of (b) after [7])

\subsection{Ternary attractive co-segregation.}

Unambiguous experimental evidence for this hypothesis has been obtained in a number of systems, notably Fe-based alloys. Meticulous experiments on high purity ternary alloys have demonstrated its existence both for segregation to grain boundaries in the Fe-Ni-Sb system [21] and to the free surface in a number of Febase ternary systems [22]. ${ }^{3}$ The two substitutionals $\mathrm{Sb}$ and $\mathrm{Ni}$, far from competing for sites, enhance each other's segregation at grain boundaries (fig. 1) and the same is true for $\mathrm{Ti}$ and $\mathrm{Sb}$ at the free surface (fig. 2 ). This phenomenon, first considered as a major contribution to the mechanism of temper embrittlement in alloy steels [13,14], was subsequently questioned on the basis of a number of experiments [24-28]. However, these were on the whole limited to conditions in which these effects are actually small and are expected to be so according to the model itself, as discussed in detail elsewhere [29,1]. Unfortunately, no attempt was made to confront these results quantitatively with the equations of the model.

Similarly, B and N strongly co-segregate to the free surface of Fe-3\%Si steel, where the tight correlation between their surface concentrations, and the XPS spectra, suggest the early formation of 2-D islands of $\mathrm{BN}$ [30]. It was also demonstrated by APFIM experiments [31] that $\mathrm{P}$ and Ti strongly cosegregate to the grain boundaries of $\alpha-\mathrm{Fe}$, as they do to its free surface [22]. Here again, quite a tight correlation is observed between $\mathrm{Ti}$ and $\mathrm{P}$ coverages, both in grain boundaries and at the free surface.

More generally, it has been demonstrated in a number of ternary Fe-base systems that the surface segregation free energy of one solute varies linearly with the surface coverage of the other element (fig. 4). A similar result was obtained for grain boundaries in ternary Fe-Ni-Sb [21] as well as low-alloy steels [18] and $12 \% \mathrm{Cr}$ martensitic stainless [32] steels (fig. 5). A quite convincing although indirect confirmation of the interactive cosegregation model was that the symmetrical interaction coefficients, $\alpha_{I-M}^{\prime}$ and $\alpha^{\prime} \Phi_{-I}$, evaluated empirically through these correlations are approximately equal for most couples and are ranked in the same order as the bulk affinities of the $\mathrm{M}$ and I solutes in Fe.

\footnotetext{
3 In view of the results in fig. 1, it is really hard to understand why "the Fe-Ni-Sb system is not at all a clear example of cosegregation" [23]. In particular, if "the question is whether or not Sb segregation enhances Ni segregation", experimental evidence for this has been given for both the surface and the grain boundaries $[21,22]$
} 


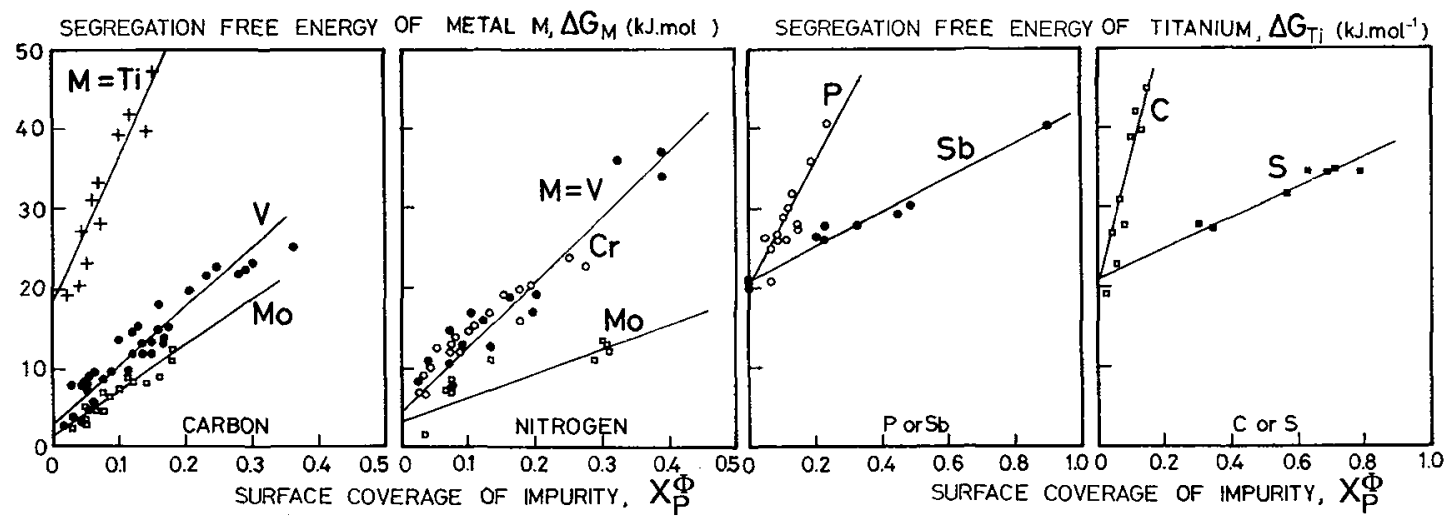

Fig. 4 -Linear correlations between segregation free energies of metallic elements ( $\mathrm{Ti}, \mathrm{Cr}, \mathrm{Mo}, \mathrm{V}$ ) and surface coverages of non-metals $(\mathrm{P}, \mathrm{Sb}, \mathrm{C}, \mathrm{N}, \mathrm{S})$ on high purity $\mathrm{Fe}$. [22]
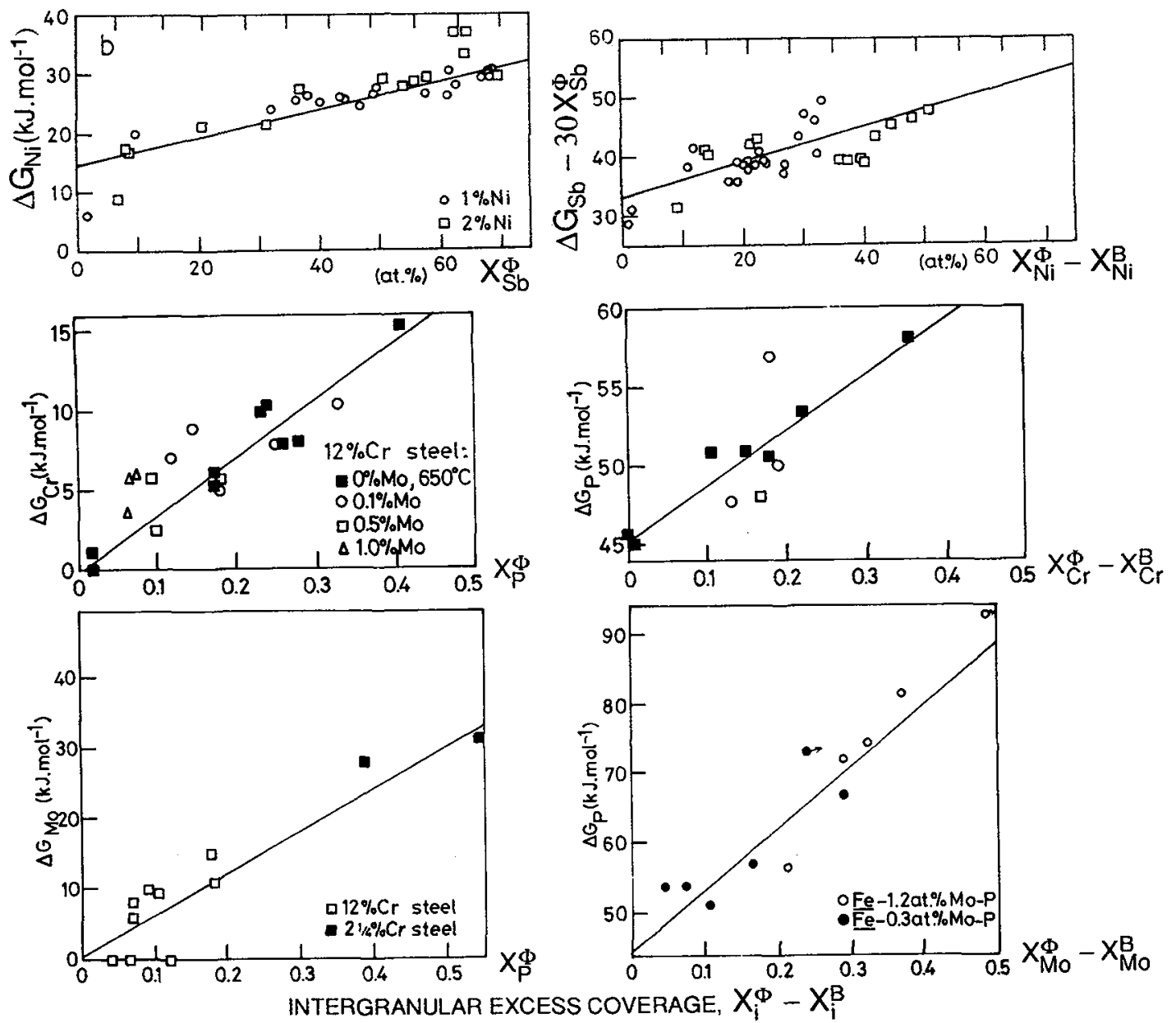

Fig. 5 - Linear correlations between segregation free energy of metallic elements and intergranular segregation of impurities, and vice-versa. a) $\mathrm{Ni}-\mathrm{Sb}[21]$; b) $\mathrm{Cr}-\mathrm{P}$ and $\mathrm{Mo}-\mathrm{P}[18,20]$ 


\subsection{The gettering effect.}

The ability of a very strong I-M interaction to eliminate impurity segregation is well documented in $\mathrm{Fe}-\mathrm{S}$ an Ni-S systems with additions of strong sulfide formers such as $\mathrm{Mn}, \mathrm{Ca}, \mathrm{Mg}, \mathrm{Zr}, \mathrm{REM}$. A spectacular illustration of the dual effect (co-segregation + gettering) of strong I-M affinity is the segregation of Ca to the free surface of $\mathrm{Ni}$ in the presence of $\mathrm{S}$ (fig. 6) [33]. Minute concentrations of Ca lead to transient cosegregation of $\mathrm{Ca}$ with $\mathrm{S}$ as long as these elements remain in supersaturation in the matrix. Upon further ageing, Ca segregation is totally supressed due to $\mathrm{CaS}$ precipitation in the matrix or at the surface, which naturally takes place earlier the higher the temperature. The overshoot in S segregation above the saturation level corresponding to the Ni-S binary is a striking illustration of cosegregation, probably associated in this case to the transient formation of a 2-D Ca sulfide at the surface. Higher $\mathrm{Ca}$ (or $\mathrm{Mg}$, Hf, etc.) additions in turn suppress $\mathrm{S}$ segregation by eliminating it altogether from the solid solution. The same is true of course for $B$ and $N$. Similarly, $T i$ which strongly cosegregates with $P$ at low concentrations efficiently traps it at higher ones [34]. Transient segregation of $P$ at the free surface of a Ti-IF steel also vanishes upon further ageing. In addition to the antagonistic effect of $\mathrm{S}$ segregation, this is due in part to the strong Ti-P interaction in the matrix, either by pair formation or phosphide precipitation [35].

Although the Mo-P affinity is much weaker than the Ti-P one, its dual antagonistic effect (cosegregation + gettering) is clearly illustrated in fig. 7 for both grain boundary and surface segregation in Fe-Mo-P alloys. In the absence of $\mathrm{P}, \mathrm{Mo}$ does not segregate at the surface of the Fe-1.2 at.\%Mo binary. The addition

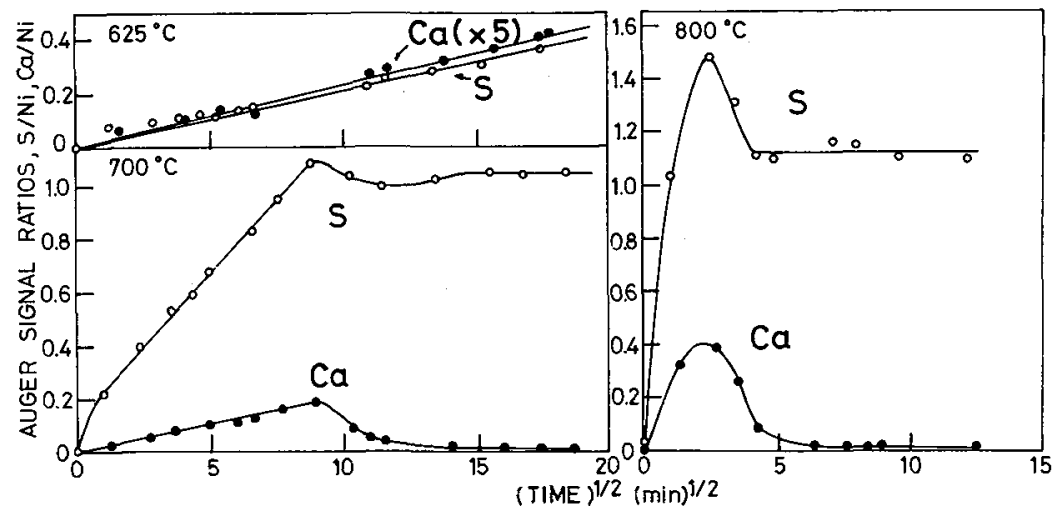

Fig. 6 - Kinetics of the cosegregation of $\mathrm{S}$ and $\mathrm{Ca}$ at the free surface of pure $\mathrm{Ni}$ at 625,700 and $800^{\circ} \mathrm{C}$. [33]
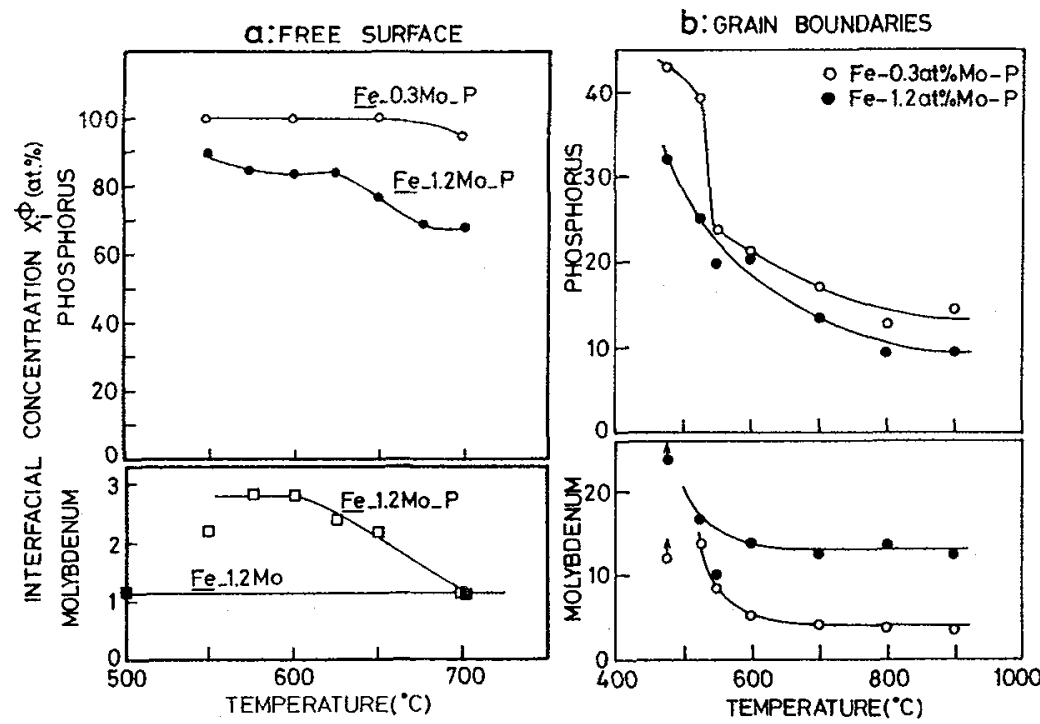

Fig. 7 - Influence of Mo and P on each other's surface and grain boundary segregation in pure Fe. [36] 
of $\mathrm{P}$ induces the segregation of Mo to the free surface, whereas the increase in Mo content from 0.3 to 1.2 at.\% decreases that of $\mathrm{P}$ by reducing its concentration in solid solution (fig. 7a). In the grain boundaries (fig. $7 \mathrm{~b}$ ), the scavenging of $\mathrm{P}$ by Mo also reduces the segregation of the impurity, especially below $550^{\circ} \mathrm{C}$, but the latter is sufficient to drive Mo to the interface as the bulk Mo content is increased.

A recent reassessment of the Mo-P affinity, which showed it to be smaller than hitherto expected, apparently invalidates this interpretation [37,38]. However, as discussed elsewhere [1], these results being obtained in alloys quenched from high temperatures, they probably largely underestimate the interactions at work below the Curie temperature, which is the range of interest for temper embrittlement and in which the Mo-P gettering effects were actually observed (see fig.7). As a matter of fact, complex multiphase particles involving $\mathrm{P}$ and Mo have been observed to form upon ageing in our Fe-P-Mo alloys [1,39]. Also, an alternative trapping mechanism of $\mathrm{P}$ atoms in the stress field of $\mathrm{Mo}_{2} \mathrm{C}$ precipitates has been observed by APFIM $[34,40]$. However, although it may be an interesting contribution in specific cases, this mechanism is not able to explain all cases of reduction in P segregation by Mo [1].

\section{REPULSIVE VERSUS COMPETITIVE SEGREGATION}

The effect of repulsive interactions between different solutes raises a basic discussion : do the observed cases of antagonistic segregation arise from repulsive interaction or from site competition?

A classical example is the mutual exclusion between segregating $\mathrm{Si}$ and $\mathrm{C}$ at the free surface of $\mathrm{Fe}$ $[6,30]$, in utter agreement with the prediction made originally on the basis of the interactive segregation model $[13,14]$. Thanks to the classical work of Darken on the Fe-Si-C system, which showed the effect of the repulsive relative $\mathrm{Si}-\mathrm{C}$ interaction on the volume diffusion of these elements in $\mathrm{Fe}$, the observations showing a similar exclusion in the surface segregation behaviour in this system have generally been interpreted without too much reluctance in terms of the repulsive segregation pattern $[6,30,55]$. Similarly, the observed decrease in P segregation induced by the segregation of Si has been tentatively attributed to $\mathrm{Si}-\mathrm{P}$ repulsion [10], which is consistent with the thermodynamics of Fe-Si-P solutions. In spite of this evidence, however, other typical examples of segregation antagonisms remain a matter of debate.

This is the case in $\alpha$-Fe for the very strong segregants carbon, nitrogen or boron which cause the grain boundary and/or surface segregation of $\mathrm{P}$ or $\mathrm{S}$ to decrease (fig.8) $[7,8,12,41]$. This behaviour is generally interpreted in terms of site competition between the former and the latter atoms, e.g. between $\mathrm{C}$ and $\mathrm{P}$, an interpretation which ignores two facts. First, the atoms of these antagonistic species are very unlikely to occupy the same sites in all types of boundaries (see above, $\$ 2.2 .1$ ), as the ones are interstitial and the others substitutional in the matrix. Secondly, it is patent that they generally exhibit little affinity for each other and even strong repulsion in solid or even liquid Fe $[10,12,20,41]$. Also, the discrepancy at the higher coverages between the experimental data and the calculated curve based on the competition model in the case of $\mathrm{C}$ and $\mathrm{P}$ (fig. 8) indicates at least the incidence of another mechanism of incompatibility between $\mathrm{C}$ and $\mathrm{P}$ atoms at the grain boundaries. Obviously, all these considerations suggest that repulsive interactions or atomic structure effects or both are at work here. Given the well documented repulsive C-P interaction in bulk solutions, an alternative explanation based on interactive segregation with relative repulsive interactions between segregated solute atoms of these two species (i.e. $\alpha^{\prime}{ }_{\mathrm{C}} \mathrm{P}<0$ ) was shown to account for these results at least as well, provided that account were also taken of a $\mathrm{C}$-C repulsive interaction in solution (i.e. $\alpha_{C}^{\Phi}>0$ ), which is also consistent with available activity data in the solid and liquid $\mathrm{Fe}-\mathrm{C}$ system $[18,20] .{ }^{4}$ The segregation energies then read (in $\left.\mathrm{kJ} . \mathrm{mol}^{-1}\right)^{5}$ :

$$
\begin{aligned}
& \Delta \mathrm{G}_{\mathrm{P}}=55.5-8.9 \mathrm{X}_{\mathrm{P}}^{\Phi}-18.0 \mathrm{X}_{\mathrm{C}}^{\Phi} \\
& \Delta \mathrm{G}_{\mathrm{C}}=92.4-33.4 \mathrm{X}_{\mathrm{C}}^{\Phi}-18.0 \mathrm{X}_{\mathrm{P}}^{\Phi} .
\end{aligned}
$$

where the $\mathrm{X}_{\mathrm{i}}^{\Phi}$ are the ratios of sites occupied by $\mathrm{P}$ and $\mathrm{C}$ atoms in their respective intergranular "sublattices" (i.e. $X_{i}^{\Phi} \max =1$ ). As already stressed in $\S 3.1$, both types of correlations being equally successful, it is rather the likelyhood of the physical hypothesis which must prevail. In any event, the C-P antagonism is an important phenomenon, which was not taken into account in the original cosegregation modelling of temper embrittlement of steels [13-15]. However, this phenomenon is unable to account for all the major aspects of this phenomenon and does not invalidate the latter effect and its importance in RTE [1].

\footnotetext{
4 According to ref. 6, "in most cases of simultaneous segregation of nonmetal atoms, this approach (i.e. the interactive segregation model) is not applicable, since the interactions are repulsive". The ground for this claim is quite mysterious since one cannot see why both positive and negative values should not be possible for $\alpha$ ' in the original model (equ.4), and since the two cases of interactive segregation have actually been discussed and illustrated in detail from the very beginning [13-15,20]. It is equally puzzling that the existence of repulsive interactions between some nonmetals can be admitted, notably for $\mathrm{Si}-\mathrm{C}, \mathrm{Si}-\mathrm{S}$, Si-P on free surfaces $[6,55]$, and simultaneously ignored for other nonmetal couples like C-P in grain boundaries $[7,8,42,45]$.

5 The coefficients in eqns 5 , as given in ref. 18,20, were derived from fig. 8 ; but using a different calibration factor for the Auger signal of carbon. Although the use of different calibration factors changes the values of the coefficient, the fit is similar.
} 


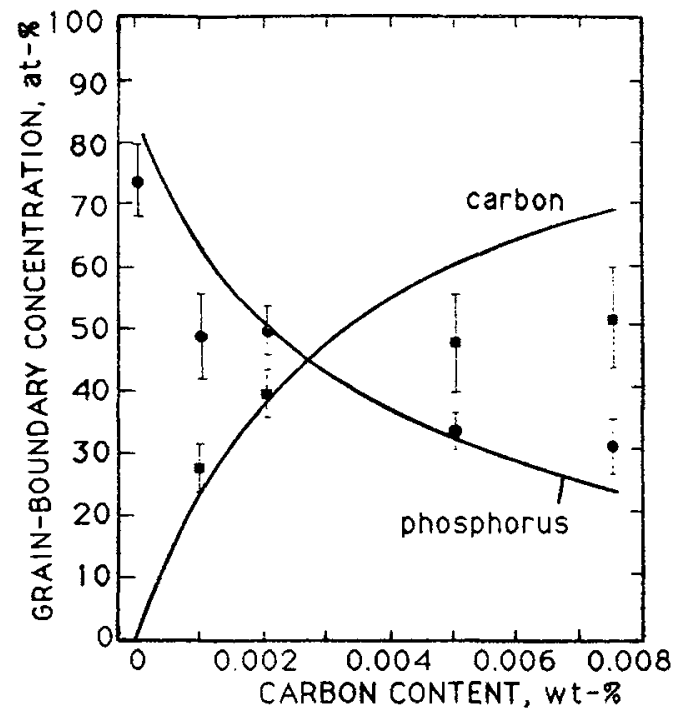

Fig. 8 - Influence of bulk $\mathrm{C}$ content on intergranular segregation of $\mathrm{P}$ and $\mathrm{C}$ in $\mathrm{Fe}$. [7]

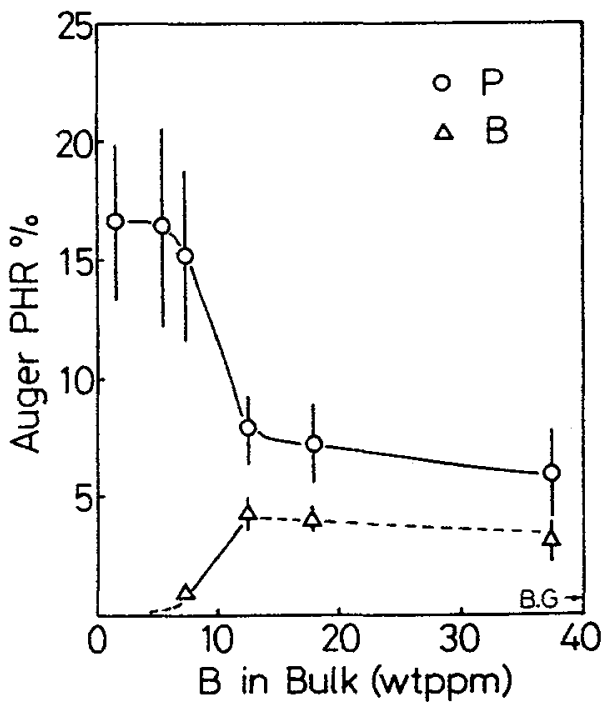

Fig. 9 - Influence of bulk B content on intergranular segregation of $\mathrm{P}$ and $\mathrm{B}$ in $\mathrm{Fe}$ at $800^{\circ} \mathrm{C}$. [41]

Quite similar segregation patterns have been observed in the Fe-Sn-C [42], Fe-S-C [12] and Fe-P-B $[9,12,43]$ systems, the intensity of the antagonism increasing in this order. The latter case is of particular scientific and technical interest, as boron exhibits an extremely high efficiency in eliminating P-induced embrittlement in $\mathrm{Fe}$ and is therefore added successfully for this purpose in some extra low carbon steels [43]. Careful experiments have shown that the intergranular segregation of $\mathrm{B}$ at $800^{\circ} \mathrm{C}$ takes place within a very narrow range of $B$ concentration in solid solution (fig. 9 , region II, $\approx 7 \mathrm{wt} p \mathrm{pm}$ wide), the first $5 \mathrm{ppm}$ or so (region I of the isotherm) being scavenged by the residual nitrogen and oxygen present in the material. Although small, B segregation leads to a considerable decrease in $P$ segregation and embrittlement. Additions in excess of $\approx 12 \mathrm{wt}$ ppm (in the alloy studied) cause B to precipitate as iron boride and its effect on $\mathrm{P}$ segregation and embrittlement to level off (region III). Due to this extremely low solubility, the $\mathrm{B}$ saturation coverage $(\approx 8$ atomic $\%)$ is much smaller than that of other segregating elements, in spite of a very high segregation energy.

The $\mathrm{P}$ - $\mathrm{B}$ antagonism has been interpreted in terms of site competition between $\mathrm{P}$ and $\mathrm{B}$. Based on the analysis of Auger peak shapes, it was concluded that no strong interaction existed between $P$ and $B$ atoms, even though it was recognised that the P-B interaction in liquid $\mathrm{Fe}$ is at least weakly repusive [41]. Given the fact that the $\mathrm{B}$ and $\mathrm{P}$ atoms are already engaged in quite strong interactions with $\mathrm{Fe}$, it is not certain that the measurement of chemical shifts in Auger spectroscopy is able to convincingly reveal the net balance of the complex relative P-B interaction pattern at the grain boundaries. Another uncanny feature which cannot be explained either with the site competition mechanism is the increase in B segregation with temperature [9]. Together with the other reservations mentioned above about the possibility of site competition between these types of atoms, it is tempting to check whether the interactive segregation rationale with repulsive P-B and P-P interactions may not account for these results as was done for the Fe-P-C system. It is probable that a repulsive B-B interaction may also have to be introduced, in analogy to the C-C one found earlier. However, not enough data are available to perform any valuable calculation. Another difficulty is that, in these results, even the $P$ segregations measured in the absence of $B$ are smaller than those found in other studies [7], which makes it impossible to use the $\Delta \mathrm{G}_{\mathrm{P}}^{\circ}$ and $\alpha_{\mathrm{P}-\mathrm{P}}^{\Phi}$ values already derived from them (see above eqns 5).

Interestingly enough, both the competition and repulsion approaches operate empirically by fitting an expression of the segregation energy like eqn $4 \mathrm{~b}$ to Auger data. The only difference lies in the opinion of the authors, which leads the competition-fans to set all the $\alpha_{i j}^{\prime}$ and $\alpha_{i j}^{\Phi}$ equal to zero and use $\Delta S_{i}^{\circ}$ as an adjustable parameter, whereas the interaction-addicts choose the reverse. In both cases, adjustable coefficients have to be introduced. Rather than in the numerical fitting of one equation or the other, the justification of either approach should rely on convincing experimental demonstration of either mechanism and on the measurement or assessment of these coefficients based on physical first principles. In the absence of these, one is left with the mere possibility of assessing the likeliness of the respective physical hypotheses. 


\section{THEORETICAL EVALUATION OF THE INTERACTIVE SEGREGATION MODEL}

The physical meaning of the chemical interaction terms in the segregation energy is quite straightforward. These terms arise quite naturally from the fact that, in general, the latter is not an ideal mixture. Thus, they build up an excess free energy of the interface. The "intrinsic" segregation energy $\Delta \mathrm{G}_{\mathrm{i}}^{\circ}$, which gives rise to very high partition ratios between the matrix and the grain boundary (sometimes of the order of thousands), causes the interface to become a concentrated 2-D solution in equilibrium with the 3-D dilute matrix. Now, in the case where appreciable chemical interactions between constituents exist, the activity of the atoms segregated in the interface, thus its excess free energy, will be strongly affected by their already high interfacial concentration. This explains that moderate interactions can have considerable effects on interfacial segregation although they do not manifest themselves very much in the bulk. This is in no way different from the case of equilibrium between dilute and concentrated 3-D phases, except that the phase rule is different when it encompasses interfaces, i.e. 2-D "phases". As demonstrated by Hillert, the 2-D interface introduces one extra degree of freedom, which can be accounted for by using the construction of the parallel instead of the common tangent in composition - free energy diagrams $[44,16]$.

"Theoretical" arguments have been opposed to the cosegregation model $[6,23,25,26,45]$. First, the thermodynamic formalism of multicomponent solutions would not apply to interfacial segregation, which would have nothing to do with bulk phases as well as with 2-D interfacial phases [26]. As this approach, "spuriously based on thermodynamics" [26], is the mere analytical extension of that of Fowler (or Defay) to 3-component systems, its soundness is in principle the same as far as basic principles are at stake !

Another physical contention was that "it is not easy to imagine the process of cosegregation" [23]. This difficulty presumably has two essential origins :

1) "One of the partner" should have "segregated before, under different conditions, by non equilibrium segregation ..." in order to be able to enhance the segregation of the other in a later, distinct process.

2) Attractively interacting elements would be unable to "diffuse to the boundary before interacting and forming, e.g., a cluster or G.P. zone in the matrix".

These naive arguments merely forget that, in general, thermodynamic systems are admitted to evolve through sequences of configurations with decreasing free energy. As stated above, the segregated interface appears as a 2-D concentrated solution where the concentrations are much larger than in the matrix from the beginning of the segregation process. Therefore, this is also the case for the excess free energies, which thus play a much more prominent role in the interface than in the 3-D dilute solution in equilibrium with it. Cosegregation can therefore be conceived, without too much imaginative strain and without resorting to complex two-step processes, in very much the same way as the innumerable well-known partition processes in multicomponent systems. The other trivial consequence of the difference in excess free energies between interface and matrix, which arises from the high intrinsic partition ratios of the elements, is that the chemical interactions and/or the bulk concentrations of at least one solute need to have comparatively high values for mutual trapping to occur in the matrix and to supersede their own amplifying effect on interfacial segregation. In this context, it should be remembered that relative interactions already explain part of the segregation behaviour in binary solutions. In the $\mathrm{Fe}-\mathrm{S}$ system for example, the strong Fe-S interaction reflected in the low solubility of sulphur, far from hindering its segregation, is on the contrary responsible for its strong intensity, in complete agreement with the well known inverse correlation between segregation and solubility established by Hondros and Seah [4]. It is therefore clear that very strongly interactive segregation can build up before any precipitation takes place. A similar reasoning can be done for the opposite case of strong solute - solute attraction.

A third argument of ten invoked is the limited validity of the regular solution model employed. The fact that its atomistic basis, i.e. pairwise interactions, is quite simplistic does not invalidate the cosegregation idea as a whole [25], since the original equations [13-15] equations merely include the general activity coefficients. We have used the regular model essentially for its analytical simplicity and for the demonstrative quality of the reasonable regular solution approximation, following the advice of M. Hillert [46]. This approximation has of ten allowed extremely valuable analyses of quite complex bulk systems [e.g. 47,48$]$. In order to release the quite restrictive hypothesis of the regular solution behaviour, the model has been subsequently extended to several other simple analytical 2-D solution models of the interface, using both a classical and a statistical thermodynamic approach : all of them basically predicted the same types of behaviours [15]. From the formal point of view, it should be noticed that the general expression of the segregation energies $\Delta \mathrm{G}_{\mathrm{i}}$ in the ternary regular solution model of interactive multiple segregation, eqn $4 \mathrm{~b}$ (table 1 ), is nothing else than the first order development of $\Delta \mathrm{G}_{\mathrm{i}}$. The regular approximation has thus the advantage of conferring a simple physical meaning on the $\alpha_{i j}$ coefficients, which allows the qualitative understanding and prediction of the behaviour of various systems on the basis of their bulk thermodynamic properties. On the other hand, not even the sign of the temperature coefficient in $\Delta \mathrm{G}_{i}$ can be simply predicted on a physical basis yet. In other words, the segregation entropy $\Delta \mathrm{S}_{\mathrm{i}}$ remains an adjustable parameter. This is another drawback of the method which identifies the segregation energies by taking into 
account the temperature coefficients only $[6,7,8,42,45]$, and neglects the more significant chemical interaction terms, as already discussed elsewhere [20].

It must be admitted, however, that the quasi-chemical model is very simplistic on one point. It identifies the grain boundary to a disordered solid solution whose structure would not depend on the nature and concentrations of the segregated species, which is obviously not true. It was expected [16] and demonstrated by numerical simulations [11] that the structure of segregated boundaries in binary alloys, like that of free surfaces, tends to assume specific atomic arrangements (akin to those encountered in 3D compounds), which depend both on the initial grain boundary structure and on the segregation. Obviously, in ternary and multicomponent alloys, these atomic patterns cannot remain unaltered if strong attractive or repulsive interactions exist between the segregating species. These considerations imply that a proper assessment of segregation energies should be able to take into account the chemical as well as the geometric and elastic properties of the atoms present. In this respect, considerable progress is expected from numerical simulations, which have already led to very promising results [11]. In spite of its shortcomings, the thermodynamic approach encompassing the chemical interaction concept nevertheless enables us to understand and predict, although on a qualitative basis only, the occurrence of 2-D "phase transitions", and these have actually been observed, classically, on the free surface, but also in the grain boundaries of a number of systems [16].

\section{INFLUENCE OF SEGREGATED ELEMENTS ON GRAIN BOUNDARY COHESION}

Most non metallic (or semi-metallic) solutes, e.g. P, S, Se, Te, Sn, Sb, O, Bi, etc, embrittle the grain boundaries of metals. It has been observed that the impact transition temperature of iron and steels increases linearly with the intergranular concentration of the embrittling species, the correlation being generally very good. The slope of these curves can be defined as an empirical measure of the "embrittling potency" of the impurity I, EPI. Only a few elements are known to oppose grain boundary fracture. It is now well established that segregated carbon does not only decrease the segregation of embrittlers (see $\$ 4$ ), but also intrinsically reinforces grain boundaries in BCC and FCC iron, and FCC nickel. The abovediscussed study of the Fe-P-B system $[9,41]$ has unquestionably demonstrated that boron also has this ability. These and other beneficial elements can also act by opposing the embrittling effects of deleterious segregated elements.

Attempts have been made to rationalize the embrittling and reinforcing effects of non metallic solutes on grain boundary cohesion using quantum physics approaches, based on charge transfer concepts [49]. These early rule of thumb attemps could not discriminate appreciably between a highly embrittling atom like $P$ and a strongly strengthening one like $C$ [23]. Recently however, it has appeared that more rigorous ab initio calculations allow significant progress towards the understanding and prediction of the cohesion of grain boundaries containing segregated non metallic elements [50,51]. It should also be remembered that the very concept of intergranular cohesion per se is notoriously insufficient to account for intergranular fracture in metals, since the latter is determined not only by the strength of atom bonds in the interface region but also by dislocation behaviour in this region and in the adjacent crystals. All the solutes known to have an influence on intergranular fracture of $\mathrm{Fe}$ have also been shown to affect its bulk plastic behaviour in a complex manner [52]. Minute amounts of these elements interact with screw dislocations and modify the formation and mobility of kink pairs. This can either strengthen or sof ten the solid solution as a whole [52], and modify the arrangement of dislocations [53], notably in the vicinity of grain boundaries. All these effects deeply alter the ductile-brittle transition behaviour, the stress concentrations on grain boundaries and the fracture mode. It is clear that a comprehensive physical model of the effects of solutes on intergranular fracture has to take all these aspects into account. In spite of the considerable progress in this area, such an understanding is far from achieved yet, and we are still left with essentially empirical approaches to tackle the macroscopic fracture properties of complex systems.

An even more complex situation is expected in ternary and multicomponent systems containing metallic alloying elements $M$. The intrinsic effect of transition metal solutes on the intergranular brittleness of pure Fe is poorly known. Manganese seems to emerge as an intrinsic embrittler [54], which is not unexpected in view of its altogether singular electronic, magnetic and physical properties in the first transition series. However, the metallic elements have generally been considered as having only indirect effects in multicomponent systems.

A very crude approach using the above-defined concept of "embrittling potency" EPI of the impurity I has been able to show that the M elements driven to grain boundaries in temper brittle steels due to their cosegregation with the embrittlers "intrinsically" modify the embrittlement [19,36]. Mn and Ni strongly increase whereas Mo decreases EPP (fig. 10). Incidentally, this effect of Mo contributes more to the alleviation of P-induced embrittlement in low-alloy steels than its gettering effect on P [1]. The physics of these effects is of course less well understood than that of individual non-metals, but the interactive segregation mechanism provides, within its own limited framework, the clue to possible partial 
explanations. Due to the strong $\mathrm{Mo}-\mathrm{C}$ interaction, the segregation of Mo would enhance that of $\mathrm{C}$, while segregated $\mathrm{Ni}$, due to the strong $\mathrm{Ni}-\mathrm{C}$ antagonism, would reduce it. Therefore, both beneficial effects of $\mathrm{C}$ (intrinsic reinforcement of grain boundary and repulsion of $P$ ) would be simultaneously enhanced in the former case and decreased in the latter.

Fig. 10 - Influence of intergranular segregation of Mo on embrittling potency of $P$ in various low alloy steel and $12 \% \mathrm{Cr}$ temper-brittle steels. $[19,36]$

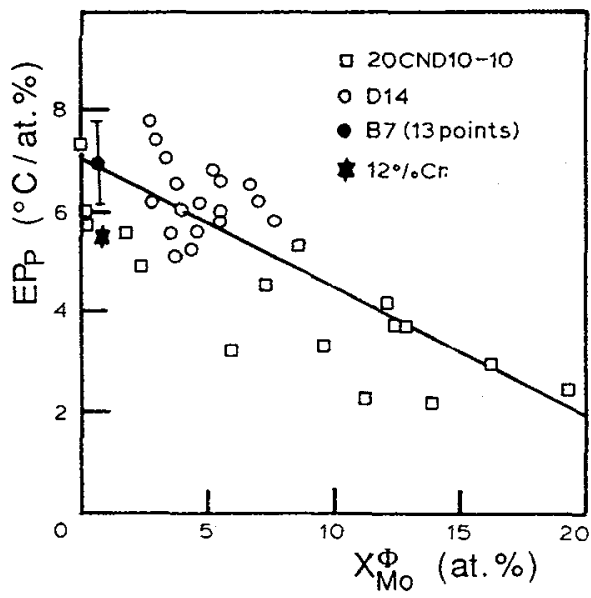

\section{CONCLUSIONS}

This brief and necessarily incomplete review of a particularly complex and controversial subject leads to the following concluding remarks :

1. Equilibrium segregation in multicomponent systems results from the intricate contribution of several different mechanisms. However, the respective ranges of validity of these mechanisms may be specific and quite narrow. They can coexist and interact or interfere to a variable extent. It is extremely difficult to discriminate between them experimentally.

2. This difficulty essentially reflects the continuing lack of precise knowledge concerning the elementary physical mechanisms which actually operate, essentially at the boundaries but also within the matrix. This ignorance arises primarily from the fact that the direct identification and measurement of the phenomena on the atomic scale in grain boundaries (interactions, competitions, etc.) is not straightforward. In the absence of such direct proofs, at least reasonable assessments of the physical significance of the various possible elementary contributions may help ... provided that they are performed without prejudice! Fortunately, the recent development of numerical simulation techniques and their application to grain boundary problems appear extremely promising.

3. The thermodynamic approach remains an efficient guiding tool for qualitatively describing and anticipating segregation behaviour, and thus for designing alternative materials solutions in order to counteract undesirable behaviours such as intergranular embrittlement. However, bare numerical correlation of segregation measurements with model equations cannot be a proof for whichever physical mechanism(s) is (are) actually operating, as the correlations with several model hypotheses often reveal equally successful at the same time. Correlations of the various models must however be attempted ... at least before declaring a given model invalid! Considerable progress can be expected from combining the numerical and the thermodynamic approaches. The former is necessary to validate the choice and combination of models, while these models allow the results of numerical calculations, necessarily limited to a small number of cases, to be genaralised to a wider range of conditions.

4. Meanwhile, interactive (attractive or repulsive) segregation remains a plausible rationale ... notably for explaining the segregation of species otherwise known to interact! The principle as well as the existence of interactive segregation and trapping effects have not been invalidated by experiments which were on the whole limited to conditions in which these effects are expected to be small according to the model itself.

5. The mechanisms of embrittlement reflect the complexity of the segregation processes, and their thorough understanding can be achieved only as far as the segregation mechanisms are understood.

\section{References}

[ 1] M. Guttmann: "Thermochemical interactions versus site competition in grain boundary segregation and embrittlement in multicomponent systems : recent literature revisited", Structural materials : engineering applications through scientific insight, NPL,Teddington, 25-26 april, 1995. (To be published)

[ 2] D. McLean: Grain Boundaries in Metals (Clarendon Press, Oxford, 1957). 
[ 3] R.H. Fowler and E.A. Guggenheim: Statistical Thermodynamics (University Press, Cambridge, 1960).

[ 4] E.D. Hondros and M.P. Seah: Scr. Metall. 6 (1972) 1007.

[ 5] R. Defay and I. Prigogine: Tension superficielle et adsorption, (Dunod, Paris, 1951).

[6] H.J. Grabke and H. Viefhaus: Surface Segregation Phenomena, chap. 8,'P.A. Dowben and A. Miller eds (CRC Press Inc., 1990) pp. 207-258.

[ 7] H. Erhart and H.J. Grabke: Metal Sci 15 (1981) 401-408.

[ 8] G. Tauber and H.J. Grabke: Ber. Bunsenges. Phys. Chem. 82 (1978) 298-302

[ 9] C.M. Liu, K. Abiko and H. Kimura: Metall. Trans. A 23A (1992) 1515-1519.

[10] C.M. Liu, K. Abiko and H. Kimura: Proc. ICSMA-8, P.O. Kettunen, T.K. Lapistö and M.E. Lehtonen eds, (Pergamon Press, New-Y ork, 1988), pp. 1101-1106.

[11] M. Hashimoto, Y. Ishida, R. Yamamoto and M. Doyama: Acta Metall. 32 (1984) 1-11.

[12] S. Suzuki, S. Tanii, K. Abiko and H. Kimura: Metall. Trans. A 18A (1987) 1109-1115.

[13] M. Guttmann: Doctoral Thesis (Université Paris XI, 1974).

[14] M. Guttmann: Surface Sci. 53 (1975) 213-227.

[15] M. Guttmann and D. McLean: Interfacial segregation, W.C. Johnson and J.M. Blakely eds (ASM, Metals Park, OH., 1979) pp. 251-348.

[16] M. Guttmann: Metall. Trans. A 8A (1977) 1383-1401.

[17] M. Guttmann: Met. Sci. 10 (1976) 337-341.

[18] M. Guttmann, P. Dumoulin and M.L. Wayman: Metall. Trans. A 13A (1982) 1693-1711.

[19] P. Dumoulin, M. Guttmann, P. Maynier and P. Chevalier: Mém. Sci. Rev. Mét. 79 (1982) 79-95 and Metal Sci. 17 (1983) 70-74.

[20] M. Guttmann: A tomistics of Fracture, R.M. Latanision and J.R. Pickens eds.(Plenum Press, New York, 1983), pp. 465-491.

[21] P. Gas, M. Guttmann and J. Bernardini: Acta Metall. 30 (1982) 1309-1316.

[22] P. Dumoulin and M. Guttmann: Mater. Sci. Eng. 42 (1980) 249-263.

[23] H.J. Grabke and C.L. Briant: Scr. Metall. 22 (1988) 1169-1172.

[24] C.L. Briant: Scr. Metall. 15 (1981) 1013-1018.

[25] C.L. Briant and A. M. Ritter: Acta Metall. 32 (1984) 2031-2042.

[26] H.J. Grabke, K. Hennesen, R. Möller and W. Wei: Scr. Metall. 21 (1987) 1329-1334.

[27] D.Y Lee, E. V. Barrera, J.P. Stark and H.L. Marcus: Metall. Trans. A 15A (1984) 1415-1430

[28] H. Kimura et al.: Proc. JIMIS-4, Suppl. to Trans. JIM 27 (1986) 53-62.

[29] J. Bernardini and P. Gas: Scr. Metall. 22 (1988) 1165-1168.

[30] L. Marchut and C.J. McMahon Jr: Metall. Trans. A 12A (1981) 1135-1139.

[31] T. Sakurai et al.: Scr. Metall. 15 (1981) 535-538.

[32] R. Guillou, M. Guttmann and P. Dumoulin: Met. Sci. 15 (1981) 63-72.

[33] A. Larère, M. Guttmann, P. Dumoulin and C. Roques-Carmes: Acta Metall. 30 (1982) 685-693.

[34] H.J. Grabke, R. Möller H. Erhart and S.S. Brenner: Surf. Interf. Anal. 10 (1987) 202-209.

[35] D. Alaoua et al.: Mater. Sci. Eng. A189 (1994) 155-163, and A174 (1994) 187-192.

[36] P. Dumoulin et al.: Metal Sci. 14 (1980) 1-15.

[37] S. Suzuki, K. Abiko and H. Kimura: Mater. Sci. Eng. 60 (1983) L17-L21.

[38] S. Suzuki, R. Hanada and H. Kimura: Mater. Sci. Eng. 80 (1986) 75-85.

[39] M.L. Wayman: Unpublished work, University of Alberta, 1981

[40] R. Möller, S.S. Brenner and H.J. Grabke: Scr. Metall. 20 (1986) 587-592.

[41] C.M. Liu, T. Nagoya, K. A biko and H. Kimura: Metall. Trans. A 23A (1992) 263-269.

[42] H.J. Grabke: ISIJ International 29 (1989) 529-538.

[43] E. Yasuhara, K. Sakata, T. Kato and O. Hashimoto: ISIJ International 34 (1994) 99-107.

[44] M. Hillert: Monograph and report series 33 (The Institute of Metals, London, 1969), pp. 231-247.

[45] H.J. Grabke: Chemistry and Physics of Fracture, R. M. Latanision and R. H. Jones eds (Martinus Nijhoff Publishers, Dordrecht, 1987), p. 23.

[46] M. Hillert: Private communication, Evry, 1973.

[47] M. Hillert: Phase transformations, chap. 5, ASM Seminars (ASM, Metals Park, OH, 1970), p. 181.

[48] M. Hillert and L.I. Staffansson: Acta Chem. Scand. 24 (1970) 3618-3628.

[49] R.P. Messmer and C.L. Briant: Acta Metall. 30 (1982) 457-467.

[50] M. Hashimoto, Y. Ishida et al.: Acta Metall. 32 (1984) 12-20 and 21-27.

[51] Ruqian Wu, A.J. Freeman and G.B. Olson: Science 265 (1994) 376-380.

[52] H. Kimura: Trans. JIM 26 (1985) 527-541 and 29 (1988) 521-539.

[53] J. Copreaux, S. Lanteri and P. Barges: Final Report, EEC Contract n ${ }^{\circ} 7210 . E C / 303,1995$.

[54] Weng Yu-King and C.J. McMahon Jr: Mater. Sci. Technol. 3 (1987) 207-216.

[55] M. Essouni; Doctoral Thesis (Université Louis Pasteur, Strasbourg, 1986). 